\title{
Optimal 3D Cell Planning: A Random Matrix Approach
}

\author{
Axel Müller*§, Jakob Hoydis ${ }^{\dagger}$, Romain Couillet**, and Mérouane Debbah ${ }^{\S}$ \\ *Intel Mobile Communications, Sophia Antipolis, France \\ ${ }^{\dagger}$ Bells Labs, Alcatel-Lucent, Stuttgart, Germany \\ ${ }^{* *}$ Department of Telecommunications and ${ }^{\S}$ Alcatel-Lucent Chair on Flexible Radio, Supélec, France \\ \{axel.mueller, romain.couillet, merouane.debbah\}@ supelec.fr, jakob.hoydis@ alcatel-lucent.com
}

\begin{abstract}
This article proposes a large system approximation of the ergodic sum-rate (SR) for cellular multi-user multipleinput multiple-output uplink systems. The considered system has various degrees of freedom, such as clusters of base stations (BSs) performing cooperative multi-point processing, randomly distributed user terminals (UTs), and supports arbitrarily configurable antenna gain patterns at the BSs. The approximation is provably tight in the limiting case of a large number of single antenna UTs and antennas at the BSs. Simulation results suggest that the asymptotic analysis is accurate for small system dimensions. Our deterministic SR approximation result is applied to numerically study and optimize the effects of antenna tilting in an exemplary sectorized 3D small cell network topology. Significant SR gains are observed with optimal tilt angles and we provide new insights on the optimal parameterization of cellular networks, along with a discussion of several non-trivial effects.
\end{abstract}

\section{INTRODUCTION}

One of the most pressing issues in designing next generation cellular networks is to support the exponentially increasing demand for wireless data-rate, given generally scarce spectrum resources. Several approaches and architectures to tackle this challenge have been investigated. One promising solution is to increase the frequency spatial reuse through cell densification. This is the main motivation for the rise of small cell networks (SCNs) [1], [2]. Base station (BS) cooperation, i.e., coordinated multipoint transmission and reception (CoMP) [3], and $3 \mathrm{D}$ beamforming (3D-BF) [4], [5] can be used to mitigate the induced inter-cell interference. A simple form of 3D-BF is antenna tilting. Antennas used for this technique can adjust the vertical tilt of their main beam, while maintaining a directive beam pattern. This shapes the physical channel by increasing the signal-to-noise ratio (SNR) for the intended user terminals (UTs), while reducing interference from non-desired UTs.

Short UT to BS distances in dense networks should result in a more pronounced tilting effect, as the change in tilting angles to focus on different users is larger compared to the case of closely co-located UTs being farther away from the BS. Shorter distances also reduce the "illuminated area" of the antennas, further enhancing the possibility to isolate UTs. Hence, sufficient support for simple vertical tilting improves interference management and increases system capacity [6]. Exploiting tilting is only possible in 3D cell planning, as the 2D approach prevalent in today's system analyses does not offer the necessary spatial degrees of freedom.
This work puts some effort in modeling the combination of SCN, CoMP, and antenna tilting in a coherent analytical framework, relying on large dimensional random matrix theory (RMT) [7], [8]. In [9], an RMT approach was presented to evaluate the sum-rate (SR) in systems with randomly distributed UTs, with the goal of optimizing BS placement. Previous work on the impact of antenna elevation angles on the ergodic mutual information of multiple-input multiple-output (MIMO) systems [10] is more centered on the antenna technology. Also, random UT placement is usually not modeled. We also mention that, in [11], a 3D system model similar to ours is used to analyze the performance of antenna tilting for almost the same topology. However, the scope of that article is on the downlink case. Furthermore, a simple matched filter precoding is assumed at the $\mathrm{BS}$, in order to derive exact performance formulas. Our work assumes instead a more involved system model, for which the exact performance analysis is intricate, hence the random matrix analysis. As such, our work allows for a more generic system analysis of the long-term benefits of antenna tilting in various cellular scenarios.

The main contributions of this paper can be summarized as follows: (i) We build upon the results in [9] and extend the deterministic SR approximation to support the modeling of clusters of cooperating BSs. We also incorporate a 3D antenna gain pattern, which approximates a standard sector antenna [4]. (ii) We exploit the results from (i) to numerically analyze and optimize the effects of antenna tilting on the achievable SR of a $\mathrm{SCN}$. Additionally, the impact of the number of BS antennas in a simple system model is considered. As opposed to standard numerical simulation tools, we show that the implementation of our equations is simple and considerably improves the simulation effort.

\section{System ModeL}

We consider an uplink multi-user MIMO (MU-MIMO) system in a 3D network topology, consisting of $K$ UTs and a number of BSs organized in $B$ clusters. Cluster $i$ consists of $B_{i}$ BSs, which act as a distributed antenna system and $K_{i}$ corresponding single-antenna UTs, where $K_{i}$ satisfies $K=\sum_{i=1}^{B} K_{i}$. The BSs within clusters can independently perform tilting. In each cluster $i$, the BSs are fully cooperating in the sense that they jointly decode the messages received from the $K_{i}$ UTs. Communication between the BSs 


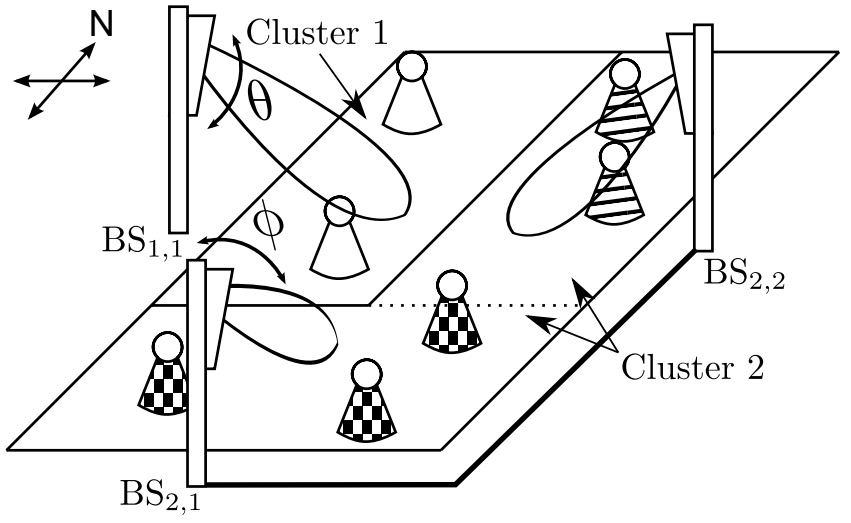

Fig. 1. Multi-cluster example for SCN deployment.

within a cluster is done through an infinite capacity backhaul link. We define $\mathrm{BS}_{i, k}, i=1, \ldots, B, k=1, \ldots, B_{i}$ as the $k$ th BS of cluster $i$. $\mathrm{BS}_{i, k}$ uses $N_{i, k}$ antennas and we denote $N_{i}=\sum_{k=1}^{B_{i}} N_{i, k}, N=\sum_{i=1}^{B} N_{i}$. BS $S_{i, k}$ has $K_{i, k}$ attached users, thus $K_{i}=\sum_{k=1}^{B_{i}} K_{i, k}$. We also denote $\mathrm{UT}_{i, k, l}$, $l=1, \ldots, K_{i, k}$, as the $l$ th UT of BS $k$ in cluster $i$. Note that, since the BSs are cooperating within a cluster, the $K_{i}$ users $\mathrm{UT}_{i, 1,1}, \ldots, \mathrm{UT}_{i, B_{i}, K_{i, B_{i}}}$ attached to cluster $i$ could have simply been indexed by $\mathrm{UT}_{i, 1}, \ldots, \mathrm{UT}_{i, K_{i}}$; however, our notation is more convenient to study the system performance for different clustering scenarios.

The placement of the BSs is arbitrary in the 3D model and $\mathrm{UT}_{i, k, l}$ is placed according to a random distribution at the position $x_{i, k, l}, i=1, \ldots, B, k=1, \ldots, B_{i}, l=1, \ldots, K_{i, k}$. An illustrative example for a possible topology with $B=$ $2, B_{1}=1, B_{2}=2, K_{1,1}=2, K_{2,1}=3$, and $K_{2,2}=2$ is shown in Figure 1. In the following, we use the indices $i$ or $j$ to identify a cluster, $k$ to identify a base station, and $l$ to identify a user; whenever this scheme is possible.

\section{A. Uplink Channel Model}

We now define the fading channel model for the interaction between the previously defined UTs and BSs. Let $\mathbf{y}_{i} \in \mathbb{C}^{N_{i}}$ be the stacked received signal vector at the BS antennas of cluster $i$. The overall received vector $\mathbf{y} \in \mathbb{C}^{N}$ of all clusters is then given by

$$
\begin{aligned}
\mathbf{y} & \triangleq\left(\begin{array}{c}
\mathbf{y}_{1} \\
\vdots \\
\mathbf{y}_{B}
\end{array}\right)=\sqrt{\rho_{\mathrm{ul}}} \mathbf{H} \mathbf{s}+\mathbf{n}=\sqrt{\rho_{\mathrm{ul}}}\left(\begin{array}{c}
\mathbf{H}_{1} \\
\vdots \\
\mathbf{H}_{B}
\end{array}\right) \mathbf{s}+\mathbf{n} \\
& =\sqrt{\rho_{\mathrm{ul}}}\left(\begin{array}{ccc}
\mathbf{H}_{1,1} & \cdots & \mathbf{H}_{1, B} \\
\vdots & \ddots & \vdots \\
\mathbf{H}_{B, 1} & \cdots & \mathbf{H}_{B, B}
\end{array}\right)\left(\begin{array}{c}
\mathbf{s}_{1} \\
\vdots \\
\mathbf{s}_{B}
\end{array}\right)+\left(\begin{array}{c}
\mathbf{n}_{1} \\
\vdots \\
\mathbf{n}_{B}
\end{array}\right)
\end{aligned}
$$

where $\mathbf{s}_{i} \sim \mathcal{C N}\left(\mathbf{0}, \mathbf{I}_{K_{i}}\right)$ is the transmit vector of all UTs in cluster $i$ and $\mathbf{s}_{i}=\left(s_{i, 1,1}, \ldots, s_{i, B_{i}, K_{i, B_{i}}}\right)^{T}$, with $s_{i, k, l}$ the transmit symbol of $\mathrm{UT}_{i, k, l}$. Here $\mathcal{C N}(\mathbf{0}, \mathbf{V})$ describes a vector valued complex Gaussian distributed random variable with zero mean and covariance matrix $\mathbf{V}$. Additionally, $\mathbf{n}_{i} \sim$
$\mathcal{C N}\left(\mathbf{0}, \mathbf{I}_{N_{i}}\right)$ is the noise vector of cluster $i$ and $\rho_{\mathrm{ul}}$ is the transmit SNR, identical for every UT. We denote $\mathbf{H}_{i, j} \in \mathbb{C}^{N_{i} \times K_{j}}$ the channel matrix from all UTs of cluster $j$ to all antennas of cluster $i$ and $\mathbf{H}_{i}=\left[\mathbf{H}_{i, 1}, \ldots, \mathbf{H}_{i, B}\right] \in \mathbb{C}^{N_{i} \times K}$. The vector channel from $\mathrm{UT}_{i, k, l}$ to the BSs of cluster $i$ is denoted $\mathbf{h}_{i, j, k, l} \in \mathbb{C}^{N_{i}}$, such that $\mathbf{H}_{i, j}=\left[\mathbf{H}_{i, j, 1}, \ldots, \mathbf{H}_{i, j, B_{j}}\right]$, where $\mathbf{H}_{i, j, k}=\left[\mathbf{h}_{i, j, k, 1}, \ldots, \mathbf{h}_{i, j, k, K_{j, k}}\right]$.

We model $\mathbf{h}_{i, j, k, l}$ as

$$
\mathbf{h}_{i, j, k, l}=\mathbf{F}_{i, j, k, l}^{\frac{1}{2}} \mathbf{g}_{i, j, k, l}
$$

where $\mathbf{g}_{i, j, k, l} \sim \mathcal{C N}\left(\mathbf{0}, \frac{1}{N} \mathbf{I}_{N_{i}}\right)$ and we define $\mathbf{F}_{i, j, k, l}=$ $\operatorname{diag}\left(f_{i, 1}\left(x_{j, k, l}\right) \mathbf{I}_{N_{i, 1}}, \ldots, f_{i, B_{i}}\left(x_{j, k, l}\right) \mathbf{I}_{N_{i, B_{i}}}\right)$.

The functions $f_{i, z}\left(x_{j, k, l}\right)$ determine the variance of the channel coefficients, i.e., $f_{i, z}\left(x_{j, k, l}\right)$ is the path loss (PL) from $\mathrm{UT}_{j, k, l}$ (at position $x_{j, k, l}$ ) with respect to $\mathrm{BS}_{i, z}$. In the following, any positive, bounded, and integrable function $f$ can be used. We take for $f$ the specific model defined in the next subsection, to allow us to model antenna tilting capabilities at the BSs.

\section{B. Pathloss Model}

The PL function used in this paper is a combination of distance dependent loss and the 3D antenna gain pattern proposed in [4]. The model presented there is a simplified 3D representation of the commonly used Kathrein 742215 antenna. It discards explicit side lobes in favor of constant gain outside the main lobe. The antenna gain $G\left(\Delta \phi_{i, z, j, k, l}, \Delta \theta_{i, z, j, k, l}\right)=$ $G(\Delta \phi, \Delta \theta)$ depends on the relative angles between the direct line from $\mathrm{UT}_{j, k, l}$ to $\mathrm{BS}_{i, z}$ and the main lobe of the antenna pattern; both in horizontal (azimuth, $\Delta \phi$ ) and vertical (tilt, $\Delta \theta)$ direction.

We denote $\Delta x_{i, z, j, k, l}=\mathrm{UT}^{x}-\mathrm{BS}^{x}=\Delta x$, where the superscript indicates the $x$-coordinate of the respective UT or BS. Similarly, $\Delta y=\mathrm{BS}^{y}-\mathrm{UT}^{y}$ and $\Delta z=\mathrm{BS}^{z}-\mathrm{UT}^{z}$. The distance $d_{i, z, j, k, l}=d$ between $\mathrm{UT}_{j, k, l}$ and $\mathrm{BS}_{i, z}$, as well as the corresponding gain $G(\Delta \phi, \Delta \theta)$ are related to the respective BS and UT positions through (3) and (6). Given the main lobe tilt $\left(\theta_{B S}\right)$ and azimuth $\left(\phi_{B S}\right)$ angle with respect to the ground and to the south orientation, respectively, and calling $\operatorname{atan} 2(y, x)$ the four-quadrant inverse tangent function, we have

$$
\begin{aligned}
d & =\sqrt{\Delta x^{2}+\Delta y^{2}+\Delta z^{2}} \\
\Delta \phi & =\operatorname{atan} 2(\Delta x, \Delta y)-\phi_{B S} \\
\Delta \theta & =\operatorname{atan} 2\left(\sqrt{\Delta x^{2}+\Delta y^{2}}, \Delta z\right)-\theta_{B S} \\
& \quad \text { with }-180^{\circ}<\theta_{B S}, \phi_{B S} \leq 180^{\circ} .
\end{aligned}
$$

In order to obtain the PL function $f$, we then express the antenna gain $G$ as follows (all " $G$-values" in Decibel):

$$
\begin{aligned}
G(\Delta \phi, \Delta \theta) & =G_{h}(\Delta \phi)+G_{v}(\Delta \theta) \quad \text { where } \\
G_{h}(\Delta \phi) & =G_{m}-\min \left\{12\left[\Delta \phi / \mathrm{HPBW}_{h}\right]^{2}, \mathrm{FBR}_{h}\right\} \\
G_{v}(\Delta \theta) & =\max \left\{-12\left[\Delta \theta / \mathrm{HPBW}_{v}\right]^{2}, \mathrm{SLL}_{v}\right\}
\end{aligned}
$$

We denote $G_{m}$ the maximum antenna gain, set to $18 \mathrm{dBi}$, $\mathrm{HPBW}_{v}$ the vertical half power beam width, set to $6.2^{\circ}$, 
$\mathrm{HPBW}_{h}$ the horizontal half power beam width, set to $65^{\circ}$, $\mathrm{FBR}_{h}$ the azimuth front-to-back ratio, set to $30 \mathrm{~dB}$, and $\mathrm{SLL}_{v}$ the tilt side lobe level, set to $-18 \mathrm{~dB}$ relative to $G_{m}$. All these values are taken in accordance with the Kathrein 742215 antenna model, which we chose due to its low complexity. Even though, it was not conceived to be used in the context of 3D$\mathrm{BF}$, but rather as a (constant tilt) sector antenna representation.

Hence, the overall PL factors contained in $\mathbf{F}_{i, j, k, l}$ are

$$
f_{i, z}\left(x_{j, k, l}\right)=10^{\frac{G\left(\Delta \phi_{i, z, j, k, l}, \Delta \theta_{i, z, j, k, l}\right)}{10}} \cdot d^{-\alpha}
$$

where $\alpha \in[2,5]$ is the PL exponent, whose value we leave unspecified for the moment. We note that $f_{i, z}\left(x_{j, k, l}\right)$ is a bounded function, as $d>0$. Further details on this section and how to obtain and motivate (7) are given in [4].

\section{Asymptotically Achievable Sum-Rate}

In this section, we study the performance of the system modeled in Section II and derive a large system approximation of the SR for a generic clustering scenario.

\section{A. Sum-Rate}

We are interested in the ergodic SR under linear detection at the receivers, i.e., the clusters of BSs, under different cooperation levels or clustering. We assume that the cooperating BSs within cluster $i$ perform a joint linear minimum-meansquare-error (MMSE) decoding on $\mathbf{y}_{i}$. Non-linear techniques, such as successive interference cancellation, at the receivers are not considered due to implementation complexity. We recall that the linear MMSE receiver maximizes the signalto-interference-plus-noise ratio (SINR).

For simplicity, we assume that all receivers have sufficient channel state information (CSI) to perform MMSE decoding. More precisely, we assume that cluster $i$ knows perfectly: (i) The matrix $\mathbf{H}_{i, i}$, or equivalently all channel vectors $\mathbf{h}_{i, i, k, l}$, and (ii) the covariance matrix $\left(\mathbf{H}_{i} \mathbf{H}_{i}^{H}+\frac{1}{\rho_{\mathrm{ul}}} \mathbf{I}_{N_{i}}\right)$. Assumption (i) is rather natural as the BSs can estimate the $\mathbf{h}_{i, i, k, l}$ based on dedicated pilot sequences sent by the UTs. The information (ii) can be obtained through approximating the covariance matrix by the sample covariance matrix of the received data. Note in particular that we do not demand that $\mathbf{H}_{i}$ is completely known at cluster $i$, which would be rather difficult to motivate.

Under these assumptions, the achievable ergodic SR normalized per UT is given by

$$
R_{\mathrm{sum}}^{\mathrm{ul}}\left(\rho_{\mathrm{ul}}\right)=\frac{1}{K} \sum_{i=1}^{B} \sum_{k=1}^{B_{i}} \sum_{l=1}^{K_{i, k}} \mathbb{E}\left[\log \left(1+\gamma_{i, k, l}^{\mathrm{ul}}\right)\right]
$$

where the expectation $(\mathbb{E})$ is taken with respect to the channels and the UT positions, and $\gamma_{i, k, l}^{\mathrm{ul}}$ is the SINR of $\mathrm{UT}_{i, k, l}$ with respect to the BSs of cluster $i$ given by

$\gamma_{i, k, l}^{\mathrm{ul}}=\mathbf{h}_{i, i, k, l}^{H}\left(\mathbf{H}_{i} \mathbf{H}_{i}^{H}-\mathbf{h}_{i, i, k, l} \mathbf{h}_{i, i, k, l}^{H}+\frac{1}{\rho_{\mathrm{ul}}} \mathbf{I}_{N_{i}}\right)^{-1} \mathbf{h}_{i, i, k, l}$.
The goal of cell planning is to optimize $R_{\text {sum }}$ with respect to variable BS parameters (e.g., placement, number and orientation of antennas). To do this one takes into account statistical information available at the BSs, such as user locations and channel statistics. This paper is particularly interested in the performance gains obtained by controlling antenna tilting. We assume here that the user positions are statistically known, i.e., $x_{j, k, l}$ is a random variable with known probability distribution, generally referred to as the UT distribution. This random UT placement poses a problem for optimization methods relying on repeated random sampling. Now both the channel and the (previously fixed) UT positions need to be repeatedly sampled, thus increasing computational complexity. An alternative to Monte-Carlo (MC) simulations, based on deterministic equivalents from random matrix theory, is proposed in the following subsection.

\section{B. Asymptotic Analysis}

In order to reduce the complexity of the optimization problem and to obtain tractable and insightful expressions of the system performance, we propose a large scale approximation. This allows us to state the SR expression in a compact form. The large scale approximation is mathematically based on the assumption that the system dimensions (number of users and number of antennas) all grow large at the same speed. Also, we need some technical assumptions in the following.

Denoting $c_{i, z}=\frac{N_{i, z}}{K}, c_{i}=\frac{N_{i}}{K}$, and $c=\frac{N}{K}$, we assume

A 1. $N_{1}, \ldots, N_{B}, K_{1}, \ldots, K_{B} \rightarrow \infty$, such that for all $i, z$ :

$$
0<\lim \sup c_{i, z} \leq \lim \sup c_{i} \leq \lim \sup c<\infty .
$$

We also denote $N \rightarrow \infty$ the convergence regime of $\mathbf{A} \mathbf{1}$.

A 2. The variables $x_{j, k, l}$ are independent random variables with finite second order moment, identically distributed across $j$, and with distribution function $F_{j, k}$.

Adapting and applying the steps in [9] to (8) and (9) yields a large scale approximation of the ergodic SR under MMSE detection for the case of joint decoding within clusters.

Proposition 1 (Deterministic SR approximation). Assume A 1 and $\mathbf{A} \mathbf{2}$, then

$$
R_{\mathrm{sum}}^{\mathrm{ul}}\left(\rho_{\mathrm{ul}}\right) \underset{N \rightarrow \infty}{\longrightarrow} \bar{R}_{\mathrm{sum}}^{\mathrm{ul}}\left(\rho_{\mathrm{ul}}\right)
$$

where

$$
\bar{R}_{\text {sum }}^{\mathrm{ul}}\left(\rho_{\mathrm{ul}}\right)=\sum_{i=1}^{B} \sum_{k=1}^{B_{i}} \frac{K_{i, k}}{K} \int \log \left(1+\bar{\gamma}_{i}^{\mathrm{ul}}(x)\right) d F_{i, k}(x)
$$

with

$$
\bar{\gamma}_{i}^{\mathrm{ul}}(x)=\sum_{k=1}^{B_{i}} \frac{N_{i, k}}{N} f_{i, k}(x) \Psi_{i, k}\left(\rho_{\mathrm{ul}}\right)
$$


and $\Psi_{i, k}, k=1, \ldots, B_{i}$ the unique non-negative solution to the fixed-point equation

$\Psi_{i, k}\left(\rho_{\mathrm{ul}}\right)=$

$\left(\frac{1}{\rho_{\mathrm{ul}}}+\sum_{j=1}^{B} \sum_{d=1}^{B_{j}} \frac{K_{j, d}}{K} \int \frac{\frac{1}{c} f_{i, k}(x) d F_{j, d}(x)}{1+\sum_{\tilde{k}=1}^{B_{i}} \frac{N_{i, \tilde{k}}}{N} f_{i, \tilde{k}}(x) \Psi_{i, \tilde{k}}\left(\rho_{\mathrm{ul}}\right)}\right)^{-1}$.

Sketch of proof: The start of the proof follows the outline given in [9]. We introduce the symbol $\stackrel{\text { a.s. }}{\longrightarrow}$ for almost sure convergence. Since the $\mathbf{F}_{i, i, k, l}$ are bounded in spectral norm and $\rho_{\mathrm{ul}}>0$, we have from [12, Lemmas F.4 and F.8]:

$$
\gamma_{i, k, l}^{\mathrm{ul}}-\frac{1}{K} \operatorname{tr} \mathbf{F}_{i, i, k, l}\left(\mathbf{H}_{i} \mathbf{H}_{i}^{H}+\frac{1}{\rho_{\mathrm{ul}}} \mathbf{I}_{N_{i}}\right)^{-1} \stackrel{\text { a.s. }}{\longrightarrow} 0 .
$$

Applying [12, Theorem 2.1] to the second term on the lefthand side, we obtain

$$
\gamma_{i, k, l}^{\mathrm{ul}}-\frac{1}{N} \sum_{b=1}^{B_{i}} N_{i, b} f_{i, b}\left(x_{j, k, l}\right) \Psi_{i, b}^{\circ} \stackrel{\text { a.s. }}{\longrightarrow} 0
$$

with $\Psi_{i, 1}^{\circ}\left(\rho_{\mathrm{ul}}\right), \ldots, \Psi_{i, B_{i}}^{\circ}\left(\rho_{\mathrm{ul}}\right)$ the unique non-negative solutions to

$$
\begin{aligned}
& \Psi_{i, b}^{\circ}\left(\rho_{\mathrm{ul}}\right)= \\
& \left(\frac{1}{\rho_{\mathrm{ul}}}+\sum_{\tilde{j}=1}^{B} \sum_{\tilde{k}=1}^{B_{\tilde{j}}} \sum_{\tilde{l}=1}^{K_{\tilde{j}, \tilde{k}}} \frac{\frac{1}{N} f_{i, b}\left(x_{\tilde{j}, \tilde{k}, \tilde{l}}\right)}{1+\sum_{\tilde{b}=1}^{B_{i}} \frac{N_{i, \tilde{b}}}{N} f_{i, \tilde{b}}\left(x_{\tilde{j}, \tilde{k}, \tilde{l}}\right) \Psi_{i, \tilde{b}}^{\circ}\left(\rho_{\mathrm{ul}}\right)}\right)^{-1} \cdot
\end{aligned}
$$

We then need to prove that $\Psi_{i, b}\left(\rho_{\mathrm{ul}}\right) \rightarrow \Psi_{i, b}^{\circ}\left(\rho_{\mathrm{ul}}\right)$ for all $i, b$ and all $\rho_{\mathrm{ul}}>0$. First, we need to show that $\Psi_{i, b}\left(\rho_{\mathrm{ul}}\right)$ exists and is a solution to the implicit equation (11). Then, writing the difference, one can show by standard manipulations and inequalities that

$$
\begin{aligned}
\max _{i, b} & \left|\Psi_{i, b}\left(\rho_{\mathrm{ul}}\right)-\Psi_{i, b}^{\circ}\left(\rho_{\mathrm{ul}}\right)\right| \\
& \leq C\left(\rho_{\mathrm{ul}}\right) \max _{i, b}\left|\Psi_{i, b}\left(\rho_{\mathrm{ul}}\right)-\Psi_{i, b}^{\circ}\left(\rho_{\mathrm{ul}}\right)\right|+\varepsilon_{N}
\end{aligned}
$$

for some sequence $\varepsilon_{N} \stackrel{\text { a.s. }}{\longrightarrow} 0$, as $N \rightarrow \infty$, and $C\left(\rho_{\mathrm{ul}}\right)$ a constant (independent of $N$ ) that converges to 0 as $\rho_{\mathrm{ul}} \rightarrow 0$. Therefore, taking $\rho_{\mathrm{ul}}$ in the connected set $\mathcal{B}=$ $\{x: C(x)<1 / 2\}$,

$$
\max _{i, b}\left|\Psi_{i, b}\left(\rho_{\mathrm{ul}}\right)-\Psi_{i, b}^{\circ}\left(\rho_{\mathrm{ul}}\right)\right| \leq \frac{\varepsilon_{N}}{1-C\left(\rho_{\mathrm{ul}}\right)} .
$$

We then have on $\mathcal{B}$, for all $i, b$,

$$
\Psi_{i, b}\left(\rho_{\mathrm{ul}}\right)-\Psi_{i, b}^{\circ}\left(\rho_{\mathrm{ul}}\right) \stackrel{\text { a.s. }}{\longrightarrow} 0 .
$$

We prove that both $\Psi_{i, b}^{\circ}$ and $\Psi_{i, b}^{\circ}$ are analytic functions (more precisely, they are Stieltjes transforms of negatively supported measures) on $\mathbb{C} \backslash \mathbb{R}_{+}$. Thus, we can use the Vitali convergence theorem and the identity theorem to show, that the above convergence holds for all $\rho_{\mathrm{ul}}>0$. From the convergence mapping theorem, we obtain

$$
\gamma_{i, k, l}^{\mathrm{ul}}-\bar{\gamma}_{i}^{\mathrm{ul}}\left(x_{i, k, l}\right) \stackrel{\text { a.s. }}{\longrightarrow} 0
$$

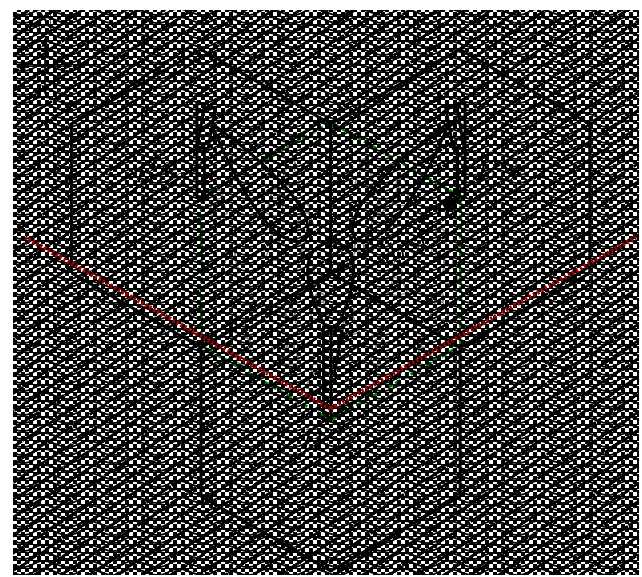

Fig. 2. Considered sectors in the 3 BS Model.

and finally

$$
R_{\mathrm{sum}}^{\mathrm{ul}}\left(\rho_{\mathrm{ul}}\right)-\bar{R}_{\mathrm{sum}}^{\mathrm{ul}}\left(\rho_{\mathrm{ul}}\right) \underset{N \rightarrow \infty}{\longrightarrow} 0
$$

which completes the proof.

This result provides an immediate approximation formula for the SR performance of large dimensional systems. Based on our notations, it is particularly easy to use Proposition 1 to evaluate the performance of various clustering scenarios. Adapting the indices of the maps between the BSs and their associated clusters, will be enough.

Although this approximation is provably valid only for large system dimensions, it happens to be accurate for small system dimensions, as well. We will provide evidence for this assertion in Section IV. Moreover, implementing Proposition 1 as a replacement for MC simulations of (8) is straightforward. The fixed-point equation converges provably and usually fast, even under simple fixed-point iteration, and the integrals can be evaluated numerically with arbitrary precision. The major advantage in terms of calculating the large scale approximation over MC simulation approaches lies in its accuracy and speed, as is evidenced in the next section.

\section{NumericAl APPLiCATION}

\section{A. Simulation Model}

We now use Proposition 1 to analyze the effect of antenna tilting on the uplink SR with MMSE detection in a sectorized cell scenario. The model consists of $3 \mathrm{BSs}$ with a height of $15 \mathrm{~m}$ arranged to form a hexagonal grid, as depicted in Figure 2. The distance between the BSs and the cell center is set to $50 \mathrm{~m}$. The azimuth angles of each BS are fixed at $180^{\circ}$ for $B S_{1}, 60^{\circ}$ for $B S_{2}$, and $-60^{\circ}$ for $B S_{3}$. All azimuth angles are given with respect to the south direction and positive values in clockwise direction, i.e., all BSs point to the cell center. Only UTs belonging to the 3 sectors in-between the BSs are considered. Taking the directivity of the antenna pattern into account this configuration already includes the strongest interferers. Tilting angles are given with respect to the ground and positive values mean upward tilting. Each 


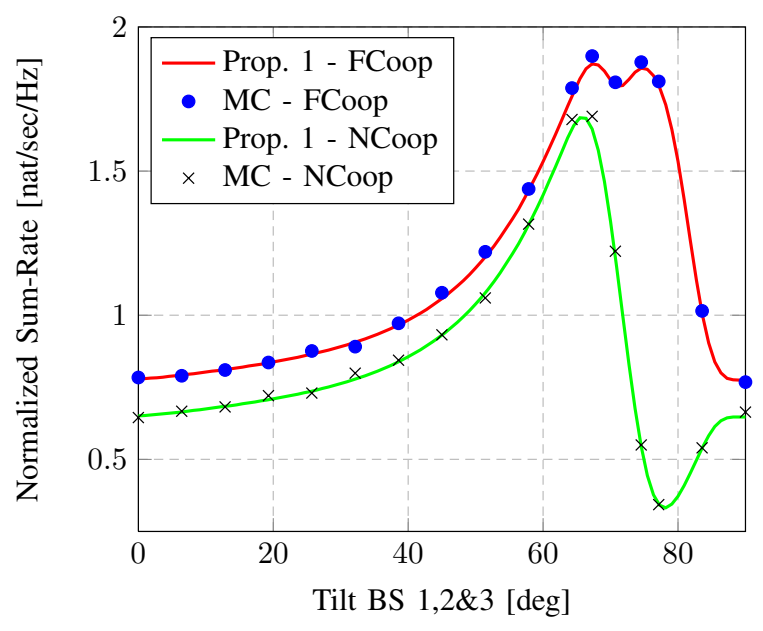

Fig. 3. SR versus tilt angle, comparison between Proposition 1 and MC simulation.

BS is equipped with 12 antennas and each sector contains 8 uniformly distributed UTs. The antenna pattern and PL model are given in Section II-B. For simplicity of interpretation, we assume that all BSs use the same tilt angle. The transmit power $\rho_{\mathrm{ul}}$ is chosen such that the received SNR from a UT located at the cell center is $5 \mathrm{~dB}$; assuming maximal antenna gain and a PL exponent of $\alpha=3.6$.

In the following, we compare two different cases of cooperation. We define the case of full cooperation (denoted FCoop) where the cell consists of a single cluster formed by all 3 BSs. The second case is that of no cooperation (denoted NCoop) in which we assume 3 clusters constituted by a single BS each.

\section{B. Optimal Tilting}

We first set the PL exponent to $\alpha=3.6$ and compare in Figure 3 the SR performance of NCoop against FCoop for different tilting angles. We provide in this figure MC simulations based on 90000 random channel realizations (300 samples of 24 UTs, each evaluated for 300 channel samples), as well as the theoretical results from Proposition 1 .

We observe that, even for these small system dimensions, the theoretical results based on Proposition 1 are extremely accurate in comparison with the MC simulations. In terms of system performance, we first see that tilting too low does not allow for sufficient users to be served. Therefore, there is a global trend, for both NCoop and FCoop, to tilt high in order to reach sufficiently many UTs. For the NCoop case, we see however that for some critical tilting angle, the interference level starts to become large; quickly reducing the SR. For the FCoop scenario, a non-straightforward behavior is observed. Two local SR maxima appear in a region above the critical tilt angle for NCoop. This is due to a trade-off between covering more UTs and, at the same time, maintaining a sufficiently low inter/intra-cluster interference level. Even in the FCoop scenario users share the same frequency resource, hence interference is the main SR limiting factor. Also, for overly high tilt angles, the closest UTs to the BSs experience

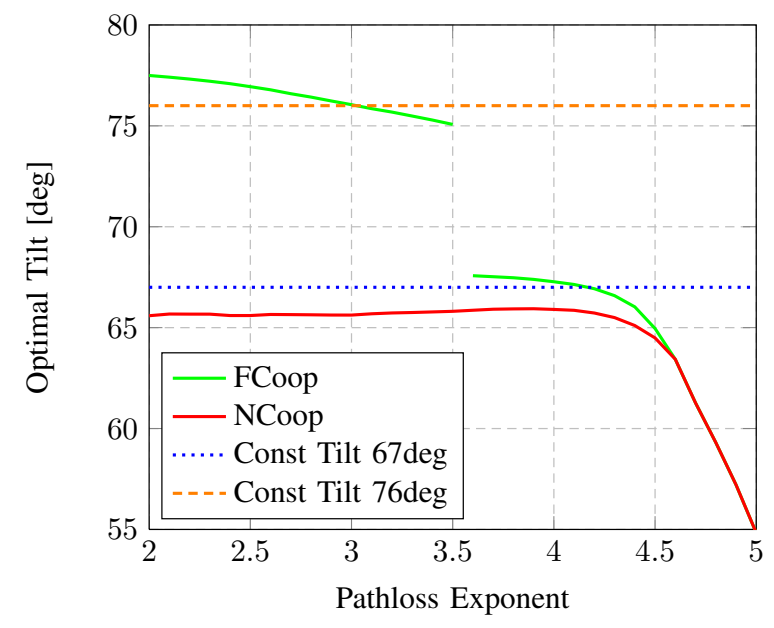

Fig. 4. Optimal tilt versus PL exponent.

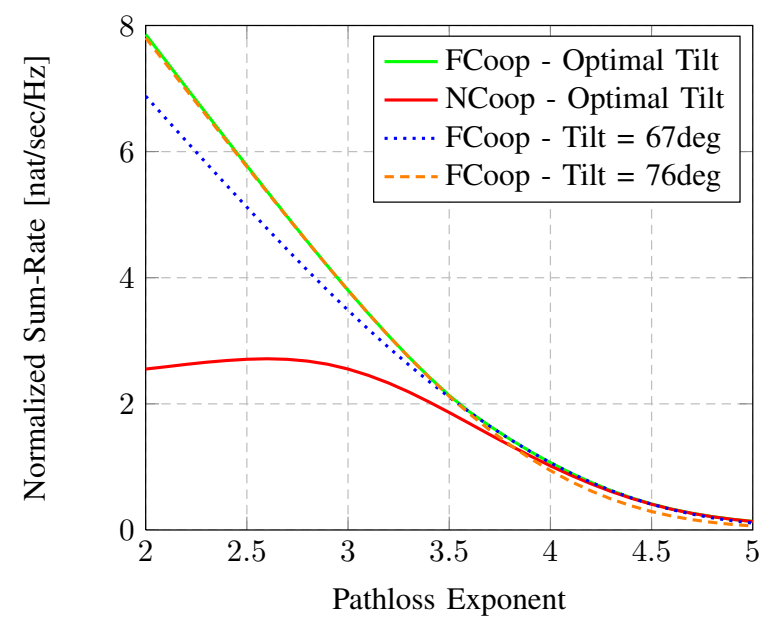

Fig. 5. SR at optimal tilt versus PL exponent.

low antenna gain and the SR dramatically drops.

In Figures 4 and 5, we focus on the influence of the PL on the optimal tilt angle and the corresponding SR. These analyses are overly computationally intensive using MC methods, thus no accuracy markers are shown. More explicitly, Figure 4 shows the impact of different PL exponents on the optimal tilting angle for both the NCoop and the FCoop scenarios. For PL exponents less than 4.5, the optimal tilt depends on the cooperation type. In the case of NCoop, the reduction of both the SNR and the interference-to-noise ratio (INR) compensate each other for varying PL, thus resulting in an almost constant optimal tilt. In the FCoop case, one observes an optimal tilt of approximately $67^{\circ}$ for $\alpha>3.6$, being consistent with the explanations above. However, a distinctive discontinuity is present around $\alpha \simeq 3.6$, where the optimal tilt angle suddenly jumps to $76^{\circ}$ for lower PL exponents. Referring back to Figure 3, this corresponds to a change in the local maximum constituting the global maximum. We now focus our attention to the normalized SR as a function of PL, which is depicted in Figure 5. One sees that, for the NCoop 


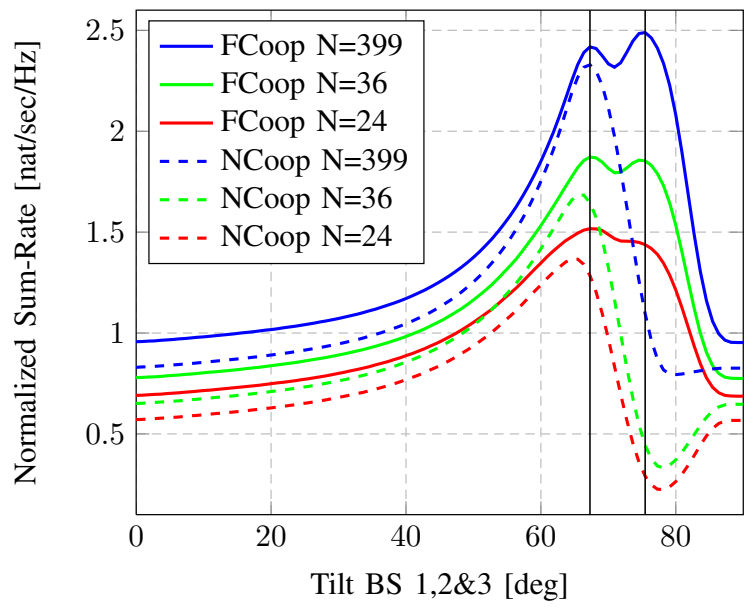

Fig. 6. SR versus tilt angle, comparison of Proposition 1 given different numbers of BS antennas.

scenario, moderate PL levels of up to 2.7 have a beneficial effect. This can be explained through the interference being attenuated for increasing PL. Higher PL exponents result in too much power loss, which over-shadows the positive effect. For very high PL exponents -larger than 3.8- we notice that intercluster interference is completely drowned out. Thus, the SR gain from optimal tilting in this regime is equivalent to the SR gain achieved by using NCoop instead of FCoop. We finally observe that non-optimal tilting, resulting from selecting the wrong local maximum in Figure 3, leads to a reduction in the achievable SR of about $10 \%$ in the FCoop scenario; both at very low and very high PL exponents. In summary we see that the optimal tilt depends in a non-obvious manner on the PL exponent and analyzing this behavior is more convenient with our proposed tools than using an MC approach.

\section{Changing the number of BS antennas}

The effect of the ratio $c$ on the SR is demonstrated by adjusting $N$ in Figure 6. Here, all BSs are assumed to have equal numbers of antennas; the other parameters are as for Figure 3. It is observed that growing $N$ also augments the $\mathrm{SR}$. However, this is only partly due to increased degrees of freedom. Additionally, the larger $N$ improves the MMSE receiver's capabilities to reject interference. Hence, one can tilt reasonably higher to increase the received signal energy and still tolerate the incurred additional (mainly inter-cluster) interference. This also changes the previously discussed local maxima balance of interference vs. signal energy, in favor of gathering more signal energy. Consequently, the rightmost maximum becomes predominant. The enhanced interference management also explains another observation: The optimal tilting angles in the NCOOP case converge to the corresponding lower local optimal tilt of the (inter-cluster interference free) FCOOP case. Finally we remark that even with very high/low numbers of BS antennas, the resulting tilting changes are too small to severely impact the SR.

\section{CONCLUSION}

We have presented a large scale approximation of the normalized ergodic SR under MMSE detection for small cell MU-MIMO uplink systems with clusters of cooperating BSs, assuming random user placement. We specifically studied the impact of antenna tilting on the resulting ergodic SR. This result was used to analyze and numerically optimize the performance of a sectorized cell scenario, assuming cooperative or non-cooperative BSs, as a function of antenna tilting. The existence of locally optimal tilts, due to a basic SNR versus interference trade-off, were observed from which insightful conclusions were drawn. We observed in particular that, for high PL scenarios, appropriate tilting can replace the SR gain brought by $\mathrm{BS}$ cooperation. This suggests that $3 \mathrm{D}-\mathrm{BF}$ is of particular interest for small cell networks.

\section{REFERENCES}

[1] J. Hoydis, M. Kobayashi, and M. Debbah, "Green small-cell networks," IEEE Vehicular Technology Magazine, vol. 6, no. 1, pp. 37-43, 2011.

[2] A. Ghosh, J. G. Andrews, N. Mangalvedhe, R. Ratasuk, B. Mondal, M. Cudak, E. Visotsky, T. A. Thomas, P. Xia, H. S. Jo, H. S. Dhillon, and T. D. Novlan, "Heterogeneous cellular networks: From theory to practice," IEEE Communications Magazine, December 2012.

[3] R. Irmer, H. Droste, P. Marsch, M. Grieger, G. Fettweis, S. Brueck, H.-P. Mayer, L. Thiele, and V. Jungnickel, "Coordinated multipoint: Concepts, performance, and field trial results," IEEE Communications Magazine, vol. 49, no. 2, pp. 102-111, 2011.

[4] F. Gunnarsson, M. N. Johansson, A. Furuskar, M. Lundevall, A. Simonsson, C. Tidestav, and M. Blomgren, "Downtilted base station antennas - a simulation model proposal and impact on HSPA and LTE performance,' in Proc. VTC 2008-Fall Vehicular Technology Conf. IEEE 68th, 2008, pp. 1-5.

[5] L. Thiele, T. Wirth, M. Schellmann, Y. Hadisusanto, and V. Jungnickel, "MU-MIMO with localized downlink base station cooperation and downtilted antennas," in Proc. IEEE Int. Conf. Communications Workshops ICC Workshops 2009, 2009, pp. 1-5.

[6] Alcatel-Lucent Shanghai Bell, "3GPP TSG-RAN WG1 \#65 R1111436: Channel modeling considerations for vertical beamforming," online, 2011, accessed 30-January-2012. [Online]. Available: http: //www.3gpp.org/ftp/tsg_ran/wg1_rl1/TSGR1_65/Docs/R1-111436.zip

[7] R. Couillet and M. Debbah, Random Matrix Methods for Wireless Communications. Cambridge University Press, November 2011.

[8] W. Hachem, P. Loubaton, and J. Najim, "Deterministic equivalents for certain functionals of large random matrices," Annals of Applied Probability, vol. 17, pp. 875-930, 2007.

[9] J. Hoydis, A. Müller, R. Couillet, and M. Debbah, "Analysis of multicell cooperation with random user locations via deterministic equivalents,' in Workshop on Spatial Stochastic Models for Wireless Networks (SpaSWiN), 2012.

[10] M. Shafi, M. Zhang, P. J. Smith, A. L. Moustakas, and A. F. Molisch, "The impact of elevation angle on MIMO capacity," in Proc. IEEE Int. Conf. Communications ICC '06, vol. 9, 2006, pp. 4155-4160.

[11] N. Seifi, M. Coldrey, and M. Viberg, "Throughput optimization in multicell MISO networks via coordinated user-specific tilting," submitted to IEEE Communication Letter, Jan. 2012.

[12] S. Wagner, "MU-MIMO transmission and reception techniques for the next generation of cellular wirelsss standards (LTE-A)," Ph.D. dissertation, EURECOM, 2229, Route des Cretes, F-06560 Sophia-Antipolis, 2011. 